\title{
Facile One-Pot Synthesis of Self-Assembled Folate-Biotin-Pullulan Nanoparticles for Targeted Intracellular Anticancer Drug Delivery
}

\author{
Ming Wang, ${ }^{1}$ Meiling Huang, ${ }^{2}$ Jianting Wang, ${ }^{2}$ Mingquan Ye, ${ }^{1}$ Yan Deng, \\ Honghao Li, ${ }^{1}$ Wensi Qian, ${ }^{1}$ Bolin Zhu, ${ }^{1}$ Yao Zhang, ${ }^{1}$ and Renmin Gong ${ }^{2}$ \\ ${ }^{1}$ Anhui Province Key Laboratory of Biological Macro-Molecules Research, Department of Biochemistry and Molecular Biology, \\ Wannan Medical College, Wuhu 241002, China \\ ${ }^{2}$ College of Life Science, Anhui Normal University, Wuhu 241000, China
}

Correspondence should be addressed to Yao Zhang; zhangyao@ahedu.gov.cn and Renmin Gong; rmgong.nju@163.com

Received 23 October 2015; Accepted 28 February 2016

Academic Editor: Ruxandra Gref

Copyright (c) 2016 Ming Wang et al. This is an open access article distributed under the Creative Commons Attribution License, which permits unrestricted use, distribution, and reproduction in any medium, provided the original work is properly cited.

\begin{abstract}
The self-assembled folate-biotin-pullulan (FBP) nanoparticles (NPs) were prepared by facile one-pot synthesis and their physicochemical properties were characterized. The self-assembled FBP NPs were used as an anticancer drug nanocarrier entrapping doxorubicin (DOX) for targeting folate-receptors-overexpressing cancer cells. The identification of prepared NPs to folate-receptor-expressing cancer cells (KB cells) was affirmed by cell viability measurement, folate competition test, and flow cytometric analysis. Compared with the naked DOX and DOX/BP NPs, the DOX/FBP NPs had lower $\mathrm{IC}_{50}$ value compared to $\mathrm{KB}$ cells as a result of the folate-receptor-mediated endocytosis process. The cytotoxicity of DOX/FBP NPs to KB cells could be inhibited competitively by free folate. The cellular intake pattern of naked DOX and drug-loaded NPs was identified by confocal laser scanning microscopy (CLSM) observation and the higher cellular uptake of drug for DOX/FBP NPs over naked DOX was observed. The prepared FBP NPs had the potential to be used as a powerful carrier to target anticancer drugs to folate-receptorexpressing tumor cells and reduce cytotoxicity to normal tissues.
\end{abstract}

\section{Introduction}

Chemotherapy is a type of cancer treatment that uses drug to destroy cancer cells. Though chemotherapy is an effective way to treat many types of cancer, it can also harm healthy cells and cause side effects. Therefore, developing a distinct carrier system that incorporates a large quantity of drug and specifically targets tumorous cells is indispensable for successful cancer chemotherapy. The application of nanotechnology in drug delivery has raised the expectations of achieving this goal and even likely changes the focus of the pharmaceutical and biotechnological industries in the future.

Polymeric nanoparticles (NPs) to be developed as anticancer drug transporters are recently emerging because of the promise in both their protection of the drug from rapid metabolism or clearance and their selective accumulation in tumorous tissues via the enhanced permeability and retention (EPR) effect caused by the vascular architecture difference of tumorous tissue and the poor lymphatic drainage system [1]. Among a variety of polymeric NPs for targeting delivery of anticancer drugs, the NPs based on natural polysaccharides, such as heparin [2], dextran [3], curdlan [4], xyloglucan [5], arabinogalactan [6], hyaluronic acid [7, 8], alginate [9-12], pullulan [13-17], and chitosan [18-25], have attracted the attention of pharmacologists. In particular, pullulan has aroused scientists' increasing interest due to its specific structure and outstanding biological properties, such as biocompatibility, biodegradability, low immunogenicity, nontoxicity, and water solubility. Pullulan is a linear polysaccharide consisting of consecutive maltotriose units, which are 
connected to each other by an $\alpha$-1,6-glycosidic bond. Pullulan has been widely used in both the food and the pharmaceutical industries.

In this study, self-assembled folate-biotin-pullulan (FBP) NPs were prepared by one-pot synthesis via DCC/DMAPmediated ester condensation. When biotin is conjugated with other biomolecules via ester or amide linkages, its water solubility dramatically decreases because of the loss of hydrophilic carboxyl group. Thus, it is expected that biotin can act as a hydrophobic moiety to form self-assembled NPs [26]. Additionally, the conjugated folate acts as a targeting ligand for specific interaction with folate receptors. These receptors can be overexpressed in the breast, ovary, endometrium, kidney, lung, head and neck, brain, and myeloid cancer cells [27-29]. The physicochemical characteristics of the prepared FBP NPs were studied using nuclear magnetic resonance (NMR), transmission electron microscopy (TEM), dynamic light scattering (DLS), electrophoretic light scattering (ELS), and fluorescence emission spectroscopy (FES). Doxorubicin (DOX), an anticancer drug commonly used in the treatment of a wide range of cancers, was encapsulated in the prepared self-assembled NPs by membrane dialysis method, and the release behavior of drug was studied by dialysis against buffer solution. The active targeting and antitumor efficiency of DOX/FBP NPs to folate-receptors-overexpressing cancer cells was investigated by cell viability measurement, folate competition test, and flow cytometric analysis. The cellular intake pattern of naked DOX and drug-loaded NPs was identified by confocal laser scanning microscopy (CLSM) observation. It is expected that the passive and active targeting ability of FBP NPs will supply a promising platform for their future application in vivo.

\section{Experimental}

2.1. Materials and Reagents. Pullulan (Mw $140 \mathrm{kDa}$ ) was obtained from Hayashibara Biochemical Laboratory, Japan. Dicyclohexyl carbodiimide (DCC), 4-dimethylaminopyridine (DMAP), pyrene, biotin, folate, 3-(4,5-dimethylthiazol2-yl)-2,5-diphenyl tetrazolium bromide (MTT, 98\%), and Hoechst 33342 were purchased from Sigma Chemical Co., USA. DOX hydrochloride (DOX $\cdot \mathrm{HCl}$ ) was acquired from Zhejiang Hisun Pharmaceutical Co. Ltd., China. KB cells (folate-receptor-overexpressing carcinoma cell line of the oral cavity) were obtained from the Fourth Military Medical University, China. RPMI 1640 growth medium without folate was purchased from Invitrogen Technologies (Carlsbad, CA). All other chemical reagents in the study were of analytical grade and obtained from commercial sources.

2.2. Synthesis of Self-Assembled NPs. The FBP was prepared by DCC/DMAP-mediated esterification reaction. Briefly, DCC/DMAP (400/200 mg) and DCC/DMAP (500/250 mg) were, respectively, added to the $70 \mathrm{~mL}$ of DMSO solution containing $0.6 \mathrm{~g}$ of folate and the $80 \mathrm{~mL}$ of DMSO solution containing $1.0 \mathrm{~g}$ of biotin with stirring for activating their carboxyl groups. Subsequently, the above two solutions were mixed and $2.0 \mathrm{~g}$ of pullulan was added with stirring. After reaction of $24 \mathrm{~h}$ at room temperature, the reactant mixture was filtered and crude product was dialyzed for removing unreacted biotin and folate against distilled water for 2 days utilizing a dialysis bag (molecular cutoff: $12 \mathrm{kDa}$ ). Distilled water was changed at intervals of $4 \mathrm{~h}$. The precipitation was recovered by filtration and washed thoroughly with diethyl ether and distilled water, followed by lyophilizing to obtain dried FBP.

The BP (without folate as targeting ligand) was synthesized by the above similar method.

The speculated one-pot synthetic route of self-assembled FBP NPs was summarized in Scheme 1.

The pullulan, synthesized BP, and FBP were analysed by ${ }^{1} \mathrm{H}$-NMR spectroscopy for confirming the biotin and folate had been incorporated into pullulan.

2.3. Preparation of Self-Assembled NPs. BP or FBP was dispersed in distilled water with gentle shaking at $37^{\circ} \mathrm{C}$ for 2 days and then ultrasonic treatment using a probe-type sonifier at $100 \mathrm{~W}$. The sonication step was repeated until the BP and FBP formed self-assembled spherical NPs. To avoid heat built up in the solution during the sonication, the pulse function (pulse on and off interval for $2.0 \mathrm{~s}$ ) was used. The solution of self-assembled NPs was then filtrated through a $1.0 \mu \mathrm{m}$ Millipore filter to remove solid impurities.

The morphology of the self-assembled FBP NPs was observed by TEM. The diameters distribution and zeta potential of prepared NPs were detected by DLS and ELS.

2.4. Fluorescence Emission Spectroscopy of FBP. The critical aggregation concentration (CAC) and self-aggregation behavior of FBP were estimated by FES using pyrene as hydrophobic fluorescent probe. The pyrene solution (1.0 $\left.\times 10^{-4} \mathrm{M}\right)$ in acetone was put into a series of test tubes, and the solution was evaporated under a nitrogen gas stream for removing acetone. Then, FBP solutions of various concentrations were introduced to the test tubes to bring the final concentration of pyrene to $6.0 \times 10^{-7} \mathrm{M}$, being roughly equal to the solubility of pyrene in water at ambient temperature. The mixture solutions were sonicated for $30 \mathrm{~min}$ in the ultrasonic bath. Pyrene emission spectra were obtained by fluorescence spectrophotometry at excitation wavelength of $343 \mathrm{~nm}$ and emission wavelength of $360-420 \mathrm{~nm}$. The intensity ratio of the third band $\left(386 \mathrm{~nm}, I_{3}\right)$ to the first band ( $374 \mathrm{~nm}, I_{1}$ ) was plotted against the logarithm of the FBP concentration. Two straight lines were drawn and the CMC value was taken from their intersection.

2.5. Preparation of DOX-Loaded NPs. DOX-loaded NPs were prepared by dialysis method. Briefly, DOX $\cdot \mathrm{HCl}(5 \mathrm{mg})$ was added to 3 equivalents of triethylamine in N,Ndimethylacetamide (DMAc) $(2 \mathrm{~mL})$ to form DOX basic adduct. Then a phosphate buffered saline (PBS) suspension containing predetermined amount of BP or FBP (100$500 \mathrm{mg}$ ) was mixed with the DOX solution and stirred overnight at $4^{\circ} \mathrm{C}$ in the dark. The mixture was then put into a dialysis bag (molecular cutoff: $12 \mathrm{kDa}$ ) and dialyzed against the PBS solution (1/15 M, pH 7.4) for 3 days at ambient temperature. The PBS solution was replaced with a fresh 

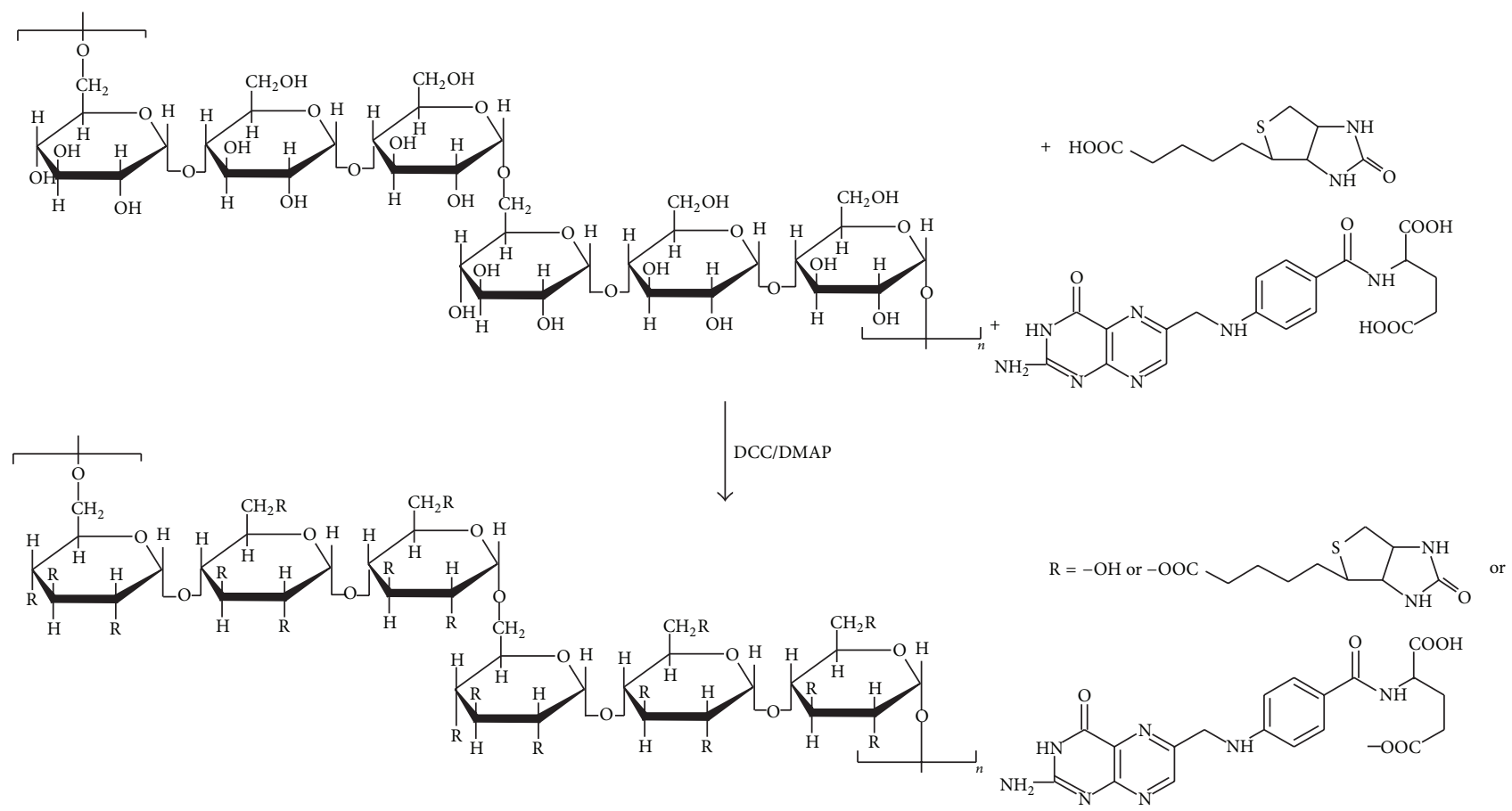

Scheme 1: One-pot synthetic route of self-assembled FBP NPs.

buffer solution every $4 \mathrm{~h}$ for the first day and then daily. The mixture was filtered through a filter $(1.0 \mu \mathrm{m}$, Millipore $)$ to remove any sedimentary polymer or drug and then lyophilized to obtain the drug-loaded NPs. The freeze-dried sample $($ carrier/drug $=40)$ was utilized for the subsequent experiments.

The thus-prepared drug-loaded sample was dissolved in DMAc and vigorously stirred for $2 \mathrm{~h}$ followed by $3 \mathrm{~min}$ of sonication. The resulting solution was centrifuged and the drug concentration in supernatant was measured by UVvis spectrophotometry at $490 \mathrm{~nm}$. The supernatant from NPs without DOX was taken as the control. The drug-loading content (LC) and loading efficiency (LE) of the NPs were calculated according to the following equations:

$$
\begin{aligned}
& \text { LC }(\%)=\frac{\text { total amount of DOX in NPs }}{\text { total amount of used NPs }} \times 100, \\
& \text { LE }(\%)=\frac{\text { total amount of DOX in NPs }}{\text { total amount of used DOX }} \times 100 .
\end{aligned}
$$

2.6. Release Profiles of DOX from Drug-Loaded NPs In Vitro. The release behavior of DOX from drug-loaded FBP NPs was studied by dialyzing against $1 / 15 \mathrm{M}$ PBS solutions at different $\mathrm{pH}$ value. Briefly, $1.5 \mathrm{~mL}$ of DOX/FBP NPs $(1 \mathrm{mg} / \mathrm{mL})$ suspension was poured onto a dialysis bag (molecular cutoff: $12 \mathrm{kDa}$ ), and the dialysis bag was submerged fully into $30 \mathrm{~mL}$ of the corresponding release medium at $37^{\circ} \mathrm{C}$ with gentle shaking. At specific time intervals, the release medium was replaced with the fresh PBS solutions. The release total amount of drug in the collected samples was measured by $\mathrm{UV}$-vis spectrophotometry at $490 \mathrm{~nm}$.
2.7. Cytotoxicity Assay. The cytotoxicities of naked DOX and two drug-loaded NPs on KB cells were determined by the MTT assay. Briefly, KB cells were cultured in folate-free RPMI 1640 culture medium supplemented with $10 \%$ fetal bovine serum, $100 \mathrm{IU} / \mathrm{mL}$ penicillin, and $100 \mu \mathrm{g} / \mathrm{mL}$ streptomycin. After incubation under a humidified atmosphere $\left(5 \% \mathrm{CO}_{2}\right.$ plus $95 \%$ air) at $37^{\circ} \mathrm{C}$ for $24 \mathrm{~h}$, the cells were inoculated into 96-well plates at a density of $1 \times 10^{4}$ cells/well and cultured in the same environment. After 1 day of growth, the cells were exposed to anticancer drug by replacing the culture medium with $100 \mu \mathrm{L}$ of freshly prepared growth medium containing various concentrations of naked DOX, DOX/BP NPs, or DOX/FBP NPs. Each DOX concentration was repeated three times and the growth medium without DOX was utilized as the control. After $48 \mathrm{~h}$ of drug exposure, the cells were washed twice with cold PBS; hereafter, $20 \mu \mathrm{L}$ of MTT dye $(5 \mathrm{mg} / \mathrm{mL}$ in PBS) was put into each well and incubated for another $4 \mathrm{~h}$ at $37^{\circ} \mathrm{C}$. The culture medium was removed and $100 \mu \mathrm{L}$ of DMSO was added to each well with vigorous shake for $10 \mathrm{~min}$ to remove any formazan crystals and then the cell viability was assessed at $570 \mathrm{~nm}$ relative to untreated cells.

2.8. Folate Competition Test. For evaluating the role of folate in the cellular uptake of drug-loaded FBP NPs, the KB cells were exposed to the DOX/FBP NPs with invariable drug concentration of $0.3 \mathrm{mg} / \mathrm{L}$ in culture medium containing different free folate concentration within the range of 0 $200 \mathrm{mg} / \mathrm{L}$. After $24 \mathrm{~h}$ incubation, the cell viability was quantified as described above. Each experiment was repeated 3 times at each folate concentration.

The competitive inhibition of folate in the cellular uptake of DOX/FBP NPs was also inspected by flow cytometric 
analysis. For flow cytometric analysis, KB cells were inoculated in 6-well plates at a density of $2 \times 10^{4}$ cells/well. After incubation for $24 \mathrm{~h}$, the culture medium in each well was substituted with fresh folate $(0.5 \mathrm{mM})$ or folate-free culture medium containing DOX/BP NPs or DOX/FBP NPs (final dose of DOX $=0.5 \mathrm{mg} / \mathrm{L}$ ). After $5 \mathrm{~h}$ of incubation, the cells were trypsinized, washed with ice-cold PBS, and stained with PI. The fluorescence intensity associated with suspended cells was analysed by the flow cytometry.

\subsection{Cellular Intake Pattern of Naked DOX and Drug-Loaded} NPs. Cellular intake pattern of naked DOX and drug-loaded NPs by KB cells was investigated by CLSM observation. Due to the inherent red fluorescence of DOX molecule, the cellular intake pattern of naked DOX and drug-loaded NPs can be observed directly by CLSM. For CLSM observation, $\mathrm{KB}$ cells at a density of $4 \times 10^{4}$ cells/well were inoculated in 6 -well plate with a piece of cover glass at the bottom of each well. After $24 \mathrm{~h}$ of incubation, the attached cells were treated with fresh folate-free culture medium containing naked DOX or drug-loaded NPs (final dose of DOX $=5 \mathrm{mg} / \mathrm{L}$ ) and incubated at standard cell culture condition for another $4 \mathrm{~h}$. The cell monolayer was then washed three times with cold PBS, fixed with $2.5 \%$ formaldehyde $(1 \mathrm{~mL} /$ well $)$ at $37^{\circ} \mathrm{C}$ for $10 \mathrm{~min}$, and then again rinsed with PBS three times. To label nucleus, fixed cells were treated with Hoechst 33342 solution $(20 \mathrm{mg} / \mathrm{mL}$ in PBS, $1 \mathrm{~mL} /$ well) for $10 \mathrm{~min}$ and then rinsed with cold PBS several times. The cover glass was placed on a glass microscope slide and visualized under CLSM in two fluorescence detection channels (red for DOX and blue for Hoechst).

2.10. Statistical Analysis. All data were presented as mean value \pm standard deviation, at least triplicate experiments. Student's $t$-test or one-way analyses of variance (ANOVA) were exercised in statistical evaluation. $P<0.05$ was considered statistically significant and $P<0.01$ was considered highly significant.

\section{Results and Discussion}

3.1. Synthesis and Characterization of Self-Assembled NPs. The synthesis of BP and FBP was confirmed by comparing their ${ }^{1} \mathrm{H}$-NMR spectra with those of the unmodified pullulan.

Figure 1 showed the ${ }^{1} \mathrm{H}$-NMR spectra of pullulan (Figure 1(a)), BP (Figure 1(b)), and FBP (Figure 1(c)). The new peaks of biotin appeared in ${ }^{1} \mathrm{H}-\mathrm{NMR}$ spectrum of BP (Figure 1(b)), such as 1.0-1.7 ppm (A, 6H, $\left.-\left(\mathrm{CH}_{2}\right)_{3}{ }^{-}, \mathrm{m}\right)$, $2.2 \mathrm{ppm}(\mathrm{B}, 2 \mathrm{H}, \mathrm{t}), 2.75 \mathrm{ppm}(\mathrm{C}, 2 \mathrm{H}, \mathrm{d}), 3.1 \mathrm{ppm}(\mathrm{D}, 1 \mathrm{H}, \mathrm{s})$, $4.1 \mathrm{ppm}(\mathrm{E}, 1 \mathrm{H}, \mathrm{dd}), 4.25 \mathrm{ppm}(\mathrm{F}, 1 \mathrm{H}, \mathrm{m})$, and $6.35 \mathrm{ppm}(\mathrm{G}$, $2 \mathrm{H},-\mathrm{NH}-, \mathrm{d}$ ), which confirmed biotin had been grafted onto the pullulan backbone successfully. The new peaks of folate appeared in ${ }^{1} \mathrm{H}$-NMR spectrum of FBP (Figure $1(\mathrm{c})$ ). The peaks at $6.6 \mathrm{ppm}$ (signal $\mathrm{H}$ ) and at $7.4 \mathrm{ppm}$ (signal I) were attributed to the protons from the benzene ring in folate and the peak at $8.6 \mathrm{ppm}$ (signal J) was assigned to proton from the pteridine ring of folate, which confirmed the successful conjugation of folate with hydroxyl groups of pullulan in BP.
TABLE 1: Characterization of prepared NPs.

\begin{tabular}{lcccc}
\hline Sample & Diameter (nm) & $\begin{array}{c}\text { Zeta potential } \\
(\mathrm{mV})\end{array}$ & LC (\%) & LE (\%) \\
\hline BP & $146 \pm 11$ & $-5.3 \pm 0.5$ & $/$ & $/$ \\
FBP & $156 \pm 13$ & $-5.1 \pm 0.6$ & $/$ & $/$ \\
DOX/FBP & $168 \pm 12$ & $-4.9 \pm 0.4$ & $1.72 \pm 0.25$ & $69 \pm 8$ \\
\hline
\end{tabular}

The TEM micrograph and diameter distribution of selfassembled FBP NPs were shown in Figure 2. The images clearly showed that the FBP NPs were spherical with average diameter of $150 \mathrm{~nm}$. The diameter of BP NPs was slightly smaller than that of FBP NPs and the drug-loaded FBP NPs exhibited an increase in diameter in comparison with the drug-free FBP NPs (Table 1).

For evaluating the stability of prepared NPs dispersed in water, the zeta potential of the NPs was investigated. The BP NPs possessed a negative surface charge and the negative surface charge decreased after conjugation of folate with positive charge groups. But the negative surface charge kept the basic stability after drug loading (Table 1). According to the previous literature reports [30-33], electrically neutral NPs often exhibited the reduced uptake rate of macrophage phagocytosis system and the prolonged circulation time in blood after systemic administration. Thus, it was deduced that FBP NPs with low surface charge could be utilized as a sustained drug delivery carrier.

The fluorescence emission (FE) spectra of pyrene in FBP solution and the CAC value of FBP were shown in Figure 3. The CAC value of FBP was $32 \mathrm{mg} / \mathrm{L}$, which was slightly higher than that $(28 \mathrm{mg} / \mathrm{L})$ reported in [26], maybe due to conjugation of folate. The low CAC value of FBP indicated that the self-assembled NPs could be formed at a reasonably low concentration because of the strong hydrophobicity of biotin moiety.

3.2. Loading and Release of DOX In Vitro. A good drug conveyer should have high LE for loaded drug; otherwise, it would cause waste of drug and limit the use of such carrier system. The FBP NPs showed relatively excellent drugloading efficiency for DOX. As shown in Figure 4(a), with the ratio of carrier/drug increasing from 20 to 100, the LE of DOX in FBP NPs increased from 63 to $86 \%$ while the LC of DOX in FBP NPs decreased from 3.15 to $0.86 \%$.

The $\mathrm{pH}$ value of normal tissues and blood is kept constantly at $\mathrm{pH}$ 7.4. However, the $\mathrm{pH}$ value of most tumorous tissues is lower than that of normal tissues. For simulating the physiological and pathological $\mathrm{pH}$ environment, three PBS solutions with different $\mathrm{pH}$ values $\mathrm{pH}$ : 7.4, 6.5, and 5.5) were used as release media to investigate the DOX release characteristic of DOX/FBP NPs. The DOX/FBP NPs exhibited the release profile relating to the $\mathrm{pH}$ value of the release environment. As shown in Figure 4(b), the DOX was released from DOX/FBP NPs very slowly at a physiological $\mathrm{pH}$ of normal tissues. Only $8.5 \%$ of DOX was released from DOX/FBP NPs at $\mathrm{pH} 7.4$ within $72 \mathrm{~h}$. In contrast, the DOX/FBP NPs exhibited significantly rapid drug release behavior at acidic $\mathrm{pH}$ value. The released total amount of 


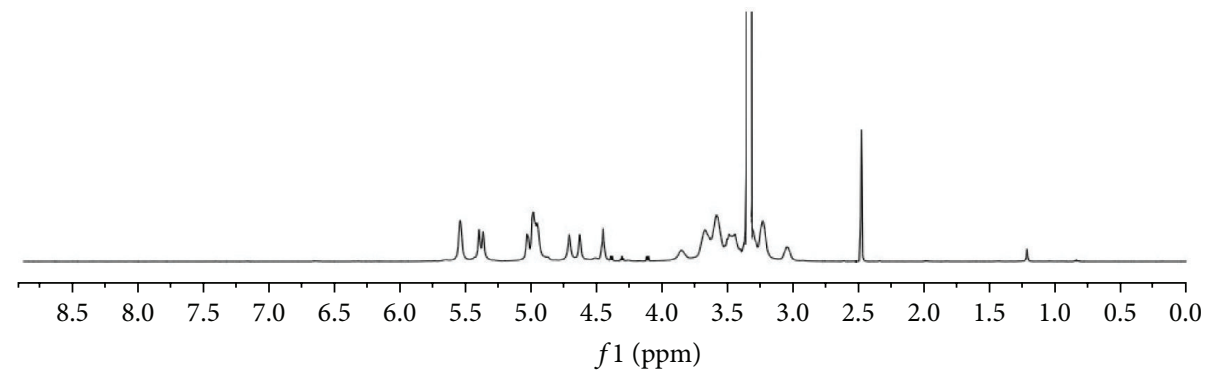

(a)

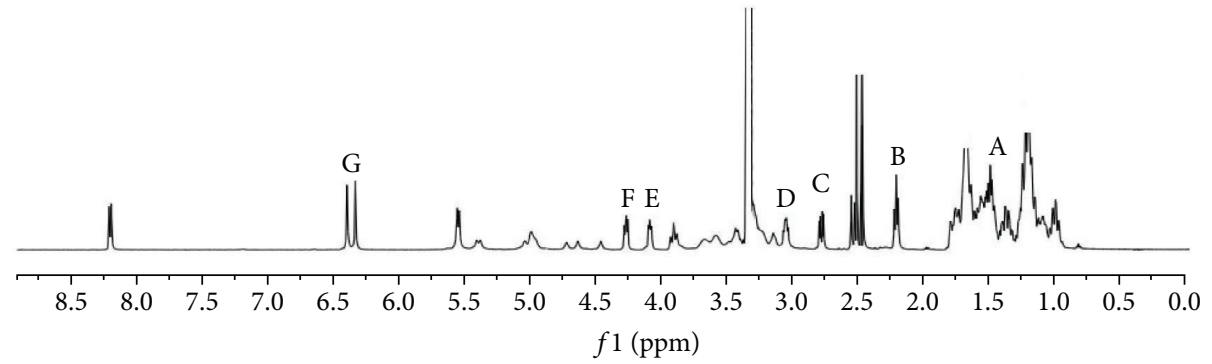

(b)

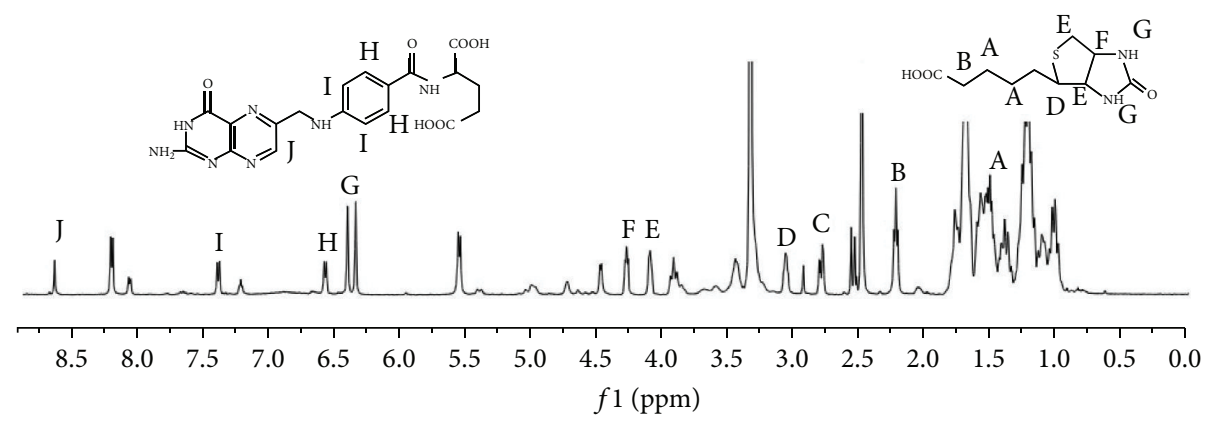

(c)

Figure 1: ${ }^{1} \mathrm{H}-\mathrm{NMR}$ spectra of pullulan (a), BP (b), and FBP (c).

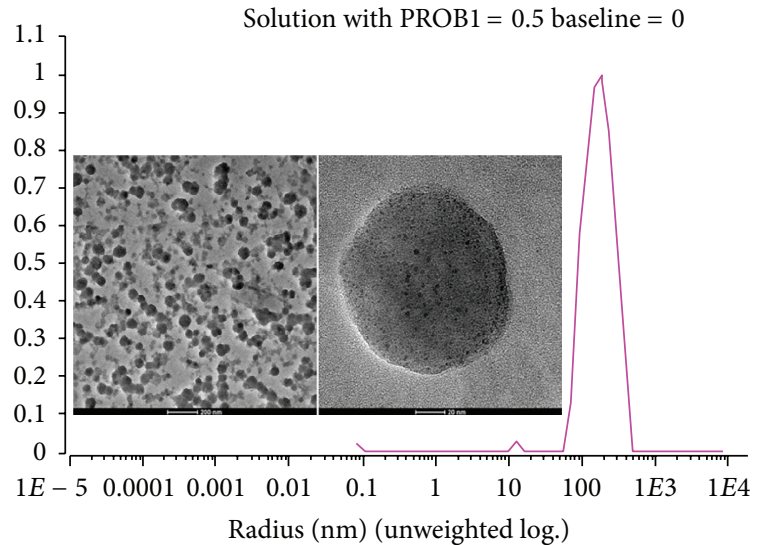

Figure 2: TEM images (scale bars: $200 \mathrm{~nm}$, left; $20 \mathrm{~nm}$, right) and diameter distribution of self-assembled FBP NPs.

DOX at pH 6.5 and $\mathrm{pH} 5.5$ was approximately 26.8 and $58.3 \%$ within $72 \mathrm{~h}$, respectively. The above results show that
DOX/FBP NPs are stable under near-neutral $\mathrm{pH}$ condition, but they deformed in acidic $\mathrm{pH}$ environments, causing the release of the enclosed drug. This drug release characteristic suggested that the FBP NPs had the potential to be utilized as drug conveyer for sustained release of hydrophobic antitumor drugs in acidic environment of cancerous tissues.

3.3. Cytotoxicity and Folate Competition Test. First, the cytotoxicities of the prepared NPs were examined and found to be relatively nontoxic up to the highest testing concentration $(500 \mathrm{mg} / \mathrm{L}$ ) against $\mathrm{KB}$ cells after 3 days of incubation (data not shown). As shown in Figure 5(a), the cytotoxicities of the naked DOX, DOX/BP NPs, and DOX/FBP NPs were enhanced with increasing of the drug concentration, showing a dose-related cytotoxicity. After 2 days of incubation, the concentration leading to $50 \%$ cell death $\left(\mathrm{IC}_{50}\right)$ of naked DOX was about $1.30 \mathrm{mg} / \mathrm{L}$. The DOX/BP NPs exhibited a slightly higher cytotoxicity than naked DOX, of which the $\mathrm{IC}_{50}$ value was about $0.75 \mathrm{mg} / \mathrm{L}$. In sharp contrast, DOX/FBP NPs were more toxic against KB cells than either naked DOX or DOX/BP NPs. The $\mathrm{IC}_{50}$ value was about $0.49 \mathrm{mg} / \mathrm{L}$, which 


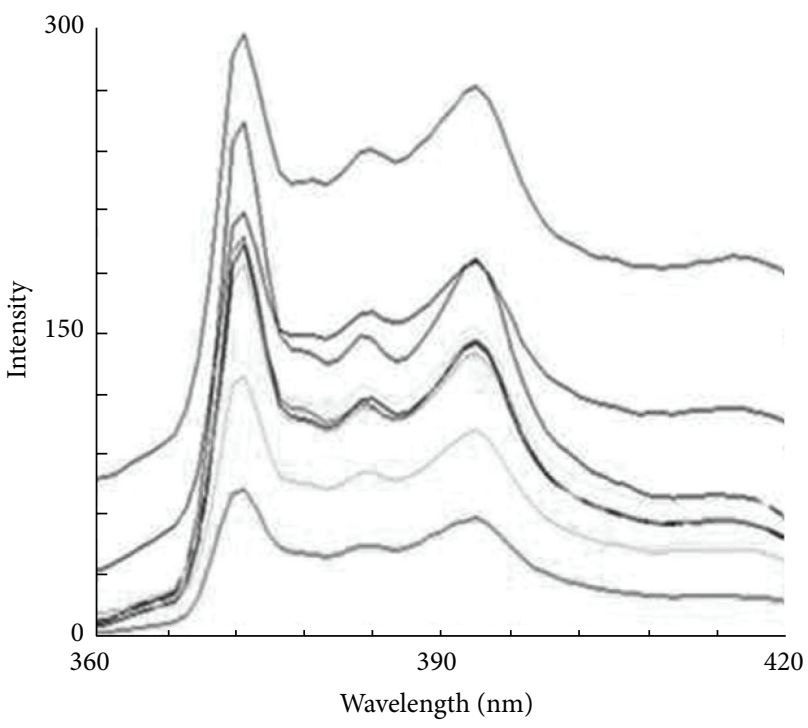

(a)

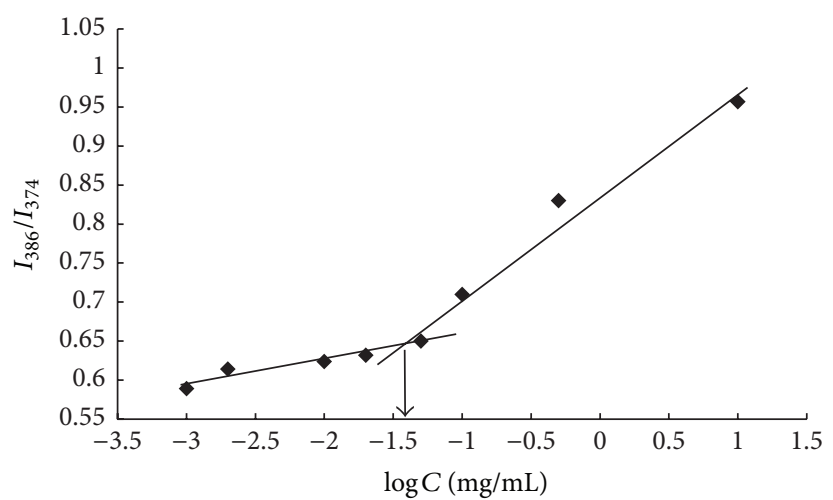

(b)

FIGURE 3: FE spectra of pyrene in FBP solutions with different concentrations (a) and intensity ratio $\left(I_{386} / I_{374}\right)$ as function of logarithm of FBP concentration (b).

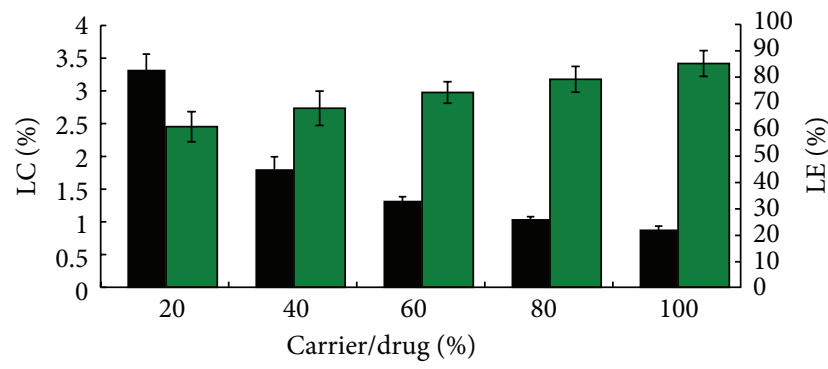

$\square \mathrm{LC}$

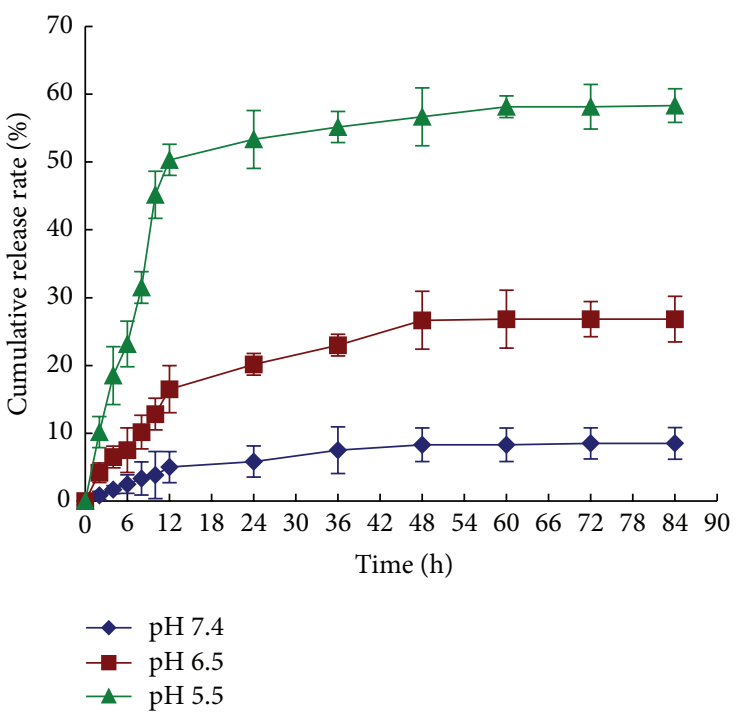

(b)

FIgURE 4: LC and LE of DOX loaded in FBP NPs (a) and release profiles of DOX from FBP NPs (b).

suggested that the folate-receptor-mediated endocytosis with targeting specificity led to larger cellular uptake of DOX and thus eliminated the tumor cells more efficiently.

The role of folate in the cellular uptake of DOX/FBP NPs was investigated by folate competition test. The cytotoxicity of DOX/FBP NPs against KB cells could be inhibited competitively by free folate, and the cell viability was improved with increasing folate concentration in culture medium (Figure 5(b)). For example, the cell viability was about $62.1 \%$ in folate-free medium but increased to about $98.3 \%$ in the presence of $200 \mathrm{mg} / \mathrm{L}$ free folate. It suggested that the free folate molecules hampered the cellular uptake of DOX/FBP
NPs by competitive combining with the folate receptors on $\mathrm{KB}$ cell surface.

The inhibiting effect of folate on the cellular uptake of DOX/FBP NPs was also evaluated by investigating the difference in apoptosis of KB cells caused by DOX/BP NPs or DOX/FBP NPs utilizing flow cytometry. As shown in Figure 6, when KB cells were incubated with DOX/BP NPs, the difference in apoptosis of $\mathrm{KB}$ cells was very little regardless of the presence or absence of folate in culture medium. However, when KB cells were incubated with DOX/FBP NPs, the enhanced difference in apoptosis of KB cells was observed in the folate-free medium compared to the folate medium. 


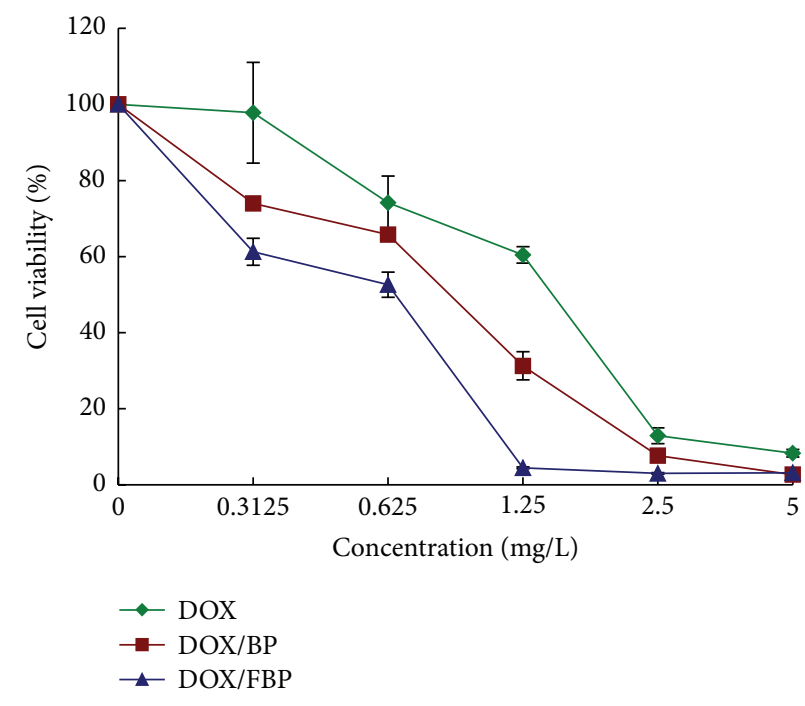

(a)

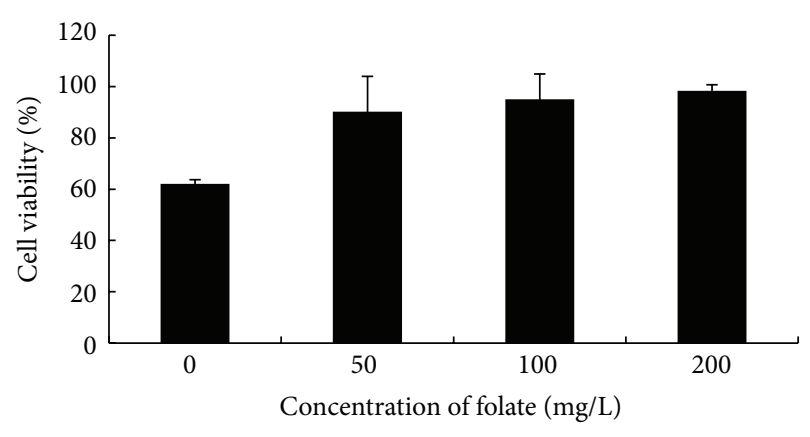

DOX/FBP

FIGURE 5: Viability of KB cells after being treated by naked DOX, DOX/BP, and DOX/FBP (a) and the effect of free folate on viability of KB cells incubated with DOX/FBP (b).

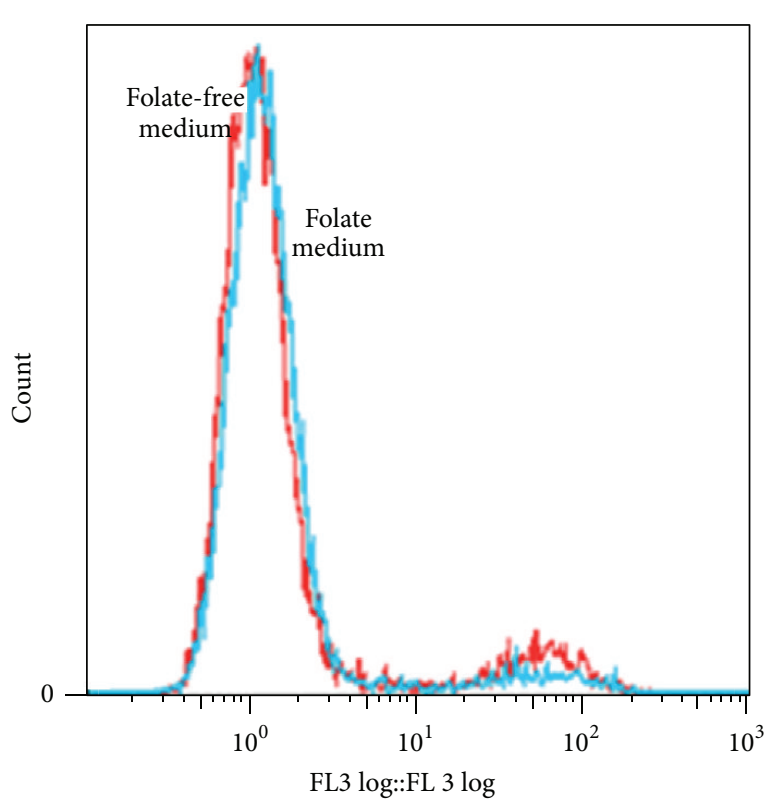

(a)

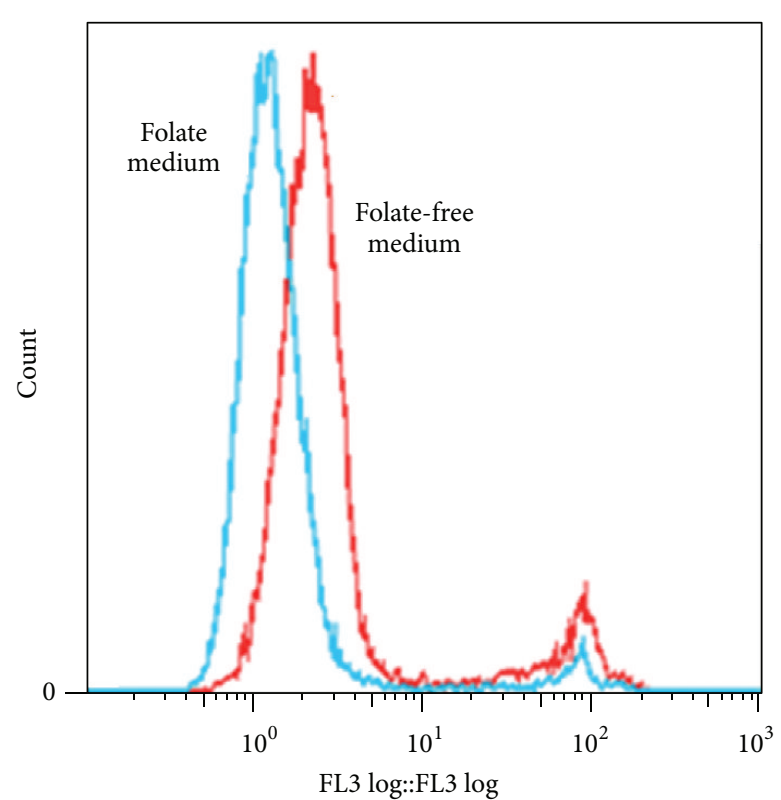

(b)

FIGURE 6: Flow cytometric analysis of DOX/BP (a) and DOX/FBP (b) in the folate medium and folate-free medium.

This indicated that free folate in the medium prevented DOX/FBP NPs from shuttling into KB cells and eliminating them by competitive combining with the folate receptors on the cell membrane.

\subsection{Cellular Intake Pattern of Naked DOX and Drug-Loaded} NPs. It is known that the free DOX molecules move into the cells by passive diffusion pattern. Because of the multidrug resistance effect, most of them are then efflux by Pglycoprotein pumps and only the drug that combined with DNA in the nucleus would remain inside the cell. The NPs are known to be absorbed into cells by endocytic pathway, thereby making them escape from the efflux action of Pglycoprotein [34].

Figure 7 showed the CLSM photos of KB cells incubated with naked DOX and DOX-loaded NPs (DOX/BP and DOX/FBP) at the same DOX concentration. The red fluorescence of DOX was utilized as probe to trace the location of drug and cell nucleus was dyed with Hoechst 33342 (blue fluorescence). After incubation with naked DOX, the DOX fluorescence located primarily in the cell nucleus with little in the cytoplasm. But, for DOX-loaded NPs, the drug 

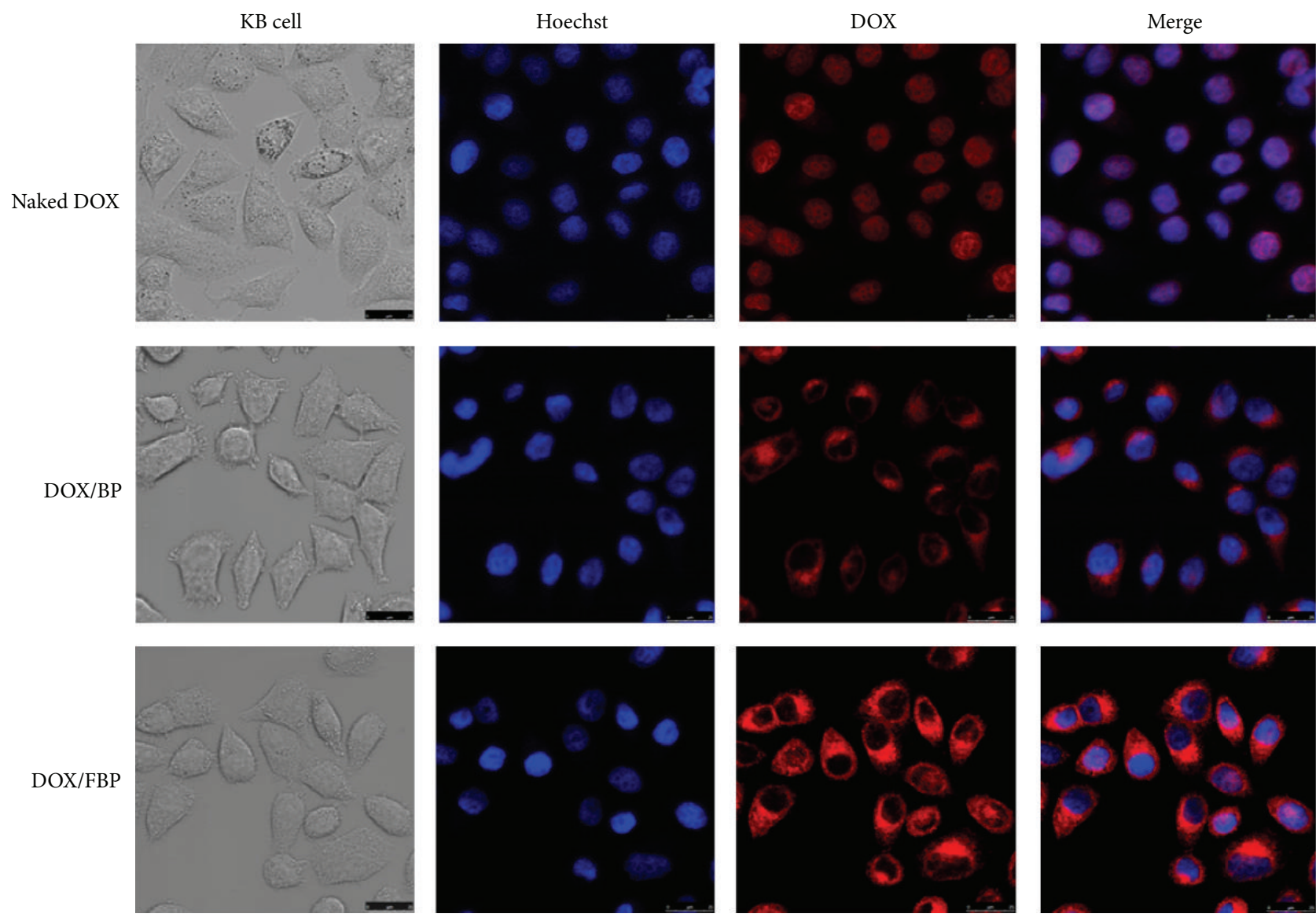

FIGURE 7: CLSM images of KB cells incubated with naked DOX, DOX/BP, and DOX/FBP NPs at the same DOX concentration for $4 \mathrm{~h}$ (scale bars: $25 \mu \mathrm{m})$.

fluorescence was observed mainly in the cytoplasm instead of the nucleus. The enhanced DOX fluorescence intensity maybe implied an increased cellular uptake at DOX/FBP NPs compared to that at naked DOX and DOX/BP NPs. It is worth noting that strong red fluorescence from DOX/FBP NPs was found on the surface of cell membrane. This suggested that the FBP NPs were absorbed into cells by the folate-receptormediated endocytosis mechanism and this mechanism might result in a higher cellular uptake. The FBP NPs inside the cells could escape the endolysosomal pathway and enter the cytoplasm where they were retained for longer time.

\section{Conclusion}

The self-assembled FBP NPs were successfully fabricated by facile method on the basis of esterification reaction and utilized to load a hydrophobic anticancer drug (DOX) with high drug-loading ability. The release of DOX from DOX/FBP NPs relied on environmental $\mathrm{pH}$ value being more rapidly in an acidic environment. This suggested that anticancer drug release could be induced by the acidic intracellular environment of tumor cells, which indicated that maybe DOX/FBP NPs could reduce the cytotoxicity to normal tissues. The DOX/FBP NPs showed increased cellular uptake, enhanced targeting ability, and reinforced cytotoxicity against KB cells.
No significant cytotoxicity was found with the prepared blank NPs themselves. Therefore, the self-assembled FBP NPs were anticipated to be a promising conveyer of hydrophobic antitumor drug for future application in vivo.

In the future, the experiments of normal cell line without folate receptor should be used as comparison to ascertain the above conclusion.

\section{Competing Interests}

The authors declare that there are no competing interests regarding the publication of this paper.

\section{Acknowledgments}

This work was supported by the Key Laboratory of Bioresource Protection and Utilization of Anhui Province, the Key Laboratory of Biotic Environment and Ecological Safety of Anhui Province, the Program for Innovative Research Team in Anhui Normal University, the Natural Science Foundation of Anhui Higher Education Institutions of China (KJ2016A728, KJ2014A266), the Foundation of Anhui Province Key Laboratory of Biological Macro-Molecules Research (1306C083008), the Independent Research Foundation of Anhui Province Key Laboratory of Biological 
Macro-Molecules Research (LAB201401, LAB201403), and the Undergraduates Innovation Project of Anhui Province (AH201310368103, AH201410368150).

\section{References}

[1] H. Maeda, J. Wu, T. Sawa, Y. Matsumura, and K. Hori, “Tumor vascular permeability and the EPR effect in macromolecular therapeutics: a review," Journal of Controlled Release, vol. 65, no. 1-2, pp. 271-284, 2000.

[2] L. Li, J. K. Kim, K. M. Huh, Y.-K. Lee, and S. Y. Kim, “Targeted delivery of paclitaxel using folate-conjugated heparinpoly( $\beta$-benzyl-l-aspartate) self-assembled nanoparticles," Carbohydrate Polymers, vol. 87, no. 3, pp. 2120-2128, 2012.

[3] B. Li, Q. Wang, X. Wang, C. Wang, and X. Jiang, "Preparation, drug release and cellular uptake of doxorubicin-loaded dextranb-poly(c-caprolactone) nanoparticles," Carbohydrate Polymers, vol. 93, no. 2, pp. 430-437, 2013.

[4] K. Na, K.-H. Park, S. W. Kim, and Y. H. Bae, "Self-assembled hydrogel nanoparticles from curdlan derivatives: characterization, anti-cancer drug release and interaction with a hepatoma cell line (HepG2)," Journal of Controlled Release, vol. 69, no. 2, pp. 225-236, 2000.

[5] Y. Cao, Y. Gu, H. Ma et al., "Self-assembled nanoparticle drug delivery systems from galactosylated polysaccharidedoxorubicin conjugate loaded doxorubicin," International Journal of Biological Macromolecules, vol. 46, no. 2, pp. 245-249, 2010.

[6] R. I. Pinhassi, Y. G. Assaraf, S. Farber et al., "Arabinogalactanfolic acid-drug conjugate for targeted delivery and targetactivated release of anticancer drugs to folate receptoroverexpressing cells," Biomacromolecules, vol. 11, no. 1, pp. 294303,2010

[7] K. Y. Choi, K. H. Min, J. H. Na et al., "Self-assembled hyaluronic acid nanoparticles as a potential drug carrier for cancer therapy: synthesis, characterization, and in vivo biodistribution," Journal of Materials Chemistry, vol. 19, no. 24, pp. 4102-4107, 2009.

[8] K. Y. Choi, H. Chung, K. H. Min et al., "Self-assembled hyaluronic acid nanoparticles for active tumor targeting," Biomaterials, vol. 31, no. 1, pp. 106-114, 2010.

[9] A. Khdair, D. Chen, Y. Patil et al., "Nanoparticle-mediated combination chemotherapy and photodynamic therapy overcomes tumor drug resistance," Journal of Controlled Release, vol. 141, no. 2, pp. 137-144, 2010.

[10] C. Zhang, W. Wang, T. Liu et al., "Doxorubicin-loaded glycyrrhetinic acid-modified alginate nanoparticles for liver tumor chemotherapy," Biomaterials, vol. 33, no. 7, pp. 2187-2196, 2012.

[11] H. Guo, Q. Lai, W. Wang et al., "Functional alginate nanoparticles for efficient intracellular release of doxorubicin and hepatoma carcinoma cell targeting therapy," International Journal of Pharmaceutics, vol. 451, no. 1-2, pp. 1-11, 2013.

[12] J. Wang, M. Wang, M. Zheng et al., "Folate mediated selfassembled phytosterol-alginate nanoparticles for targeted intracellular anticancer drug delivery," Colloids and Surfaces B: Biointerfaces, vol. 129, pp. 63-70, 2015.

[13] K. Na, E. S. Lee, and Y. H. Bae, "Adriamycin loaded pullulan acetate/sulfonamide conjugate nanoparticles responding to tumor $\mathrm{pH}$ : $\mathrm{pH}$-dependent cell interaction, internalization and cytotoxicity in vitro," Journal of Controlled Release, vol. 87, no. 1-3, pp. 3-13, 2003.
[14] Y.-I. Jeong, H.-S. Na, J.-S. Oh, K.-C. Choi, C.-E. Song, and H.C. Lee, "Adriamycin release from self-assembling nanospheres of poly(dl-lactide-co-glycolide)-grafted pullulan," International Journal of Pharmaceutics, vol. 322, no. 1-2, pp. 154-160, 2006.

[15] H.-Z. Zhang, X.-M. Li, F.-P. Gao, L.-R. Liu, Z.-M. Zhou, and Q.-Q. Zhang, "Preparation of folate-modified pullulan acetate nanoparticles for tumor-targeted drug deliveryal," Drug Delivery, vol. 17, no. 1, pp. 48-57, 2010.

[16] A. Scomparin, S. Salmaso, S. Bersani, R. Satchi-Fainaro, and P. Caliceti, "Novel folated and non-folated pullulan bioconjugates for anticancer drug delivery," European Journal of Pharmaceutical Sciences, vol. 42, no. 5, pp. 547-558, 2011.

[17] W. Yang, M. Wang, L. Ma, H. Li, and L. Huang, "Synthesis and characterization of biotin modified cholesteryl pullulan as a novel anticancer drug carrier," Carbohydrate Polymers, vol. 99, pp. 720-727, 2014.

[18] Y.-S. Wang, L.-R. Liu, Q. Jiang, and Q.-Q. Zhang, "Selfaggregated nanoparticles of cholesterol-modified chitosan conjugate as a novel carrier of epirubicin," European Polymer Journal, vol. 43, no. 1, pp. 43-51, 2007.

[19] J.-H. Kim, Y.-S. Kim, K. Park et al., "Antitumor efficacy of cisplatin-loaded glycol chitosan nanoparticles in tumor-bearing mice," Journal of Controlled Release, vol. 127, no. 1, pp. 41-49, 2008.

[20] H. F. Zhou, W. T. Yu, X. Guo et al., "Synthesis and characterization of amphiphilic glycidol-chitosan-deoxycholic acid nanoparticles as a drug carrier for doxorubicin," Biomacromolecules, vol. 11, no. 12, pp. 3480-3486, 2010.

[21] P.-P. Lv, Y.-F. Ma, R. Yu et al., "Targeted delivery of insoluble cargo (paclitaxel) by PEGylated chitosan nanoparticles grafted with Arg-Gly-Asp (RGD)," Molecular Pharmaceutics, vol. 9, no. 6, pp. 1736-1747, 2012.

[22] Y. Y. Qiu, J. Zhu, S. J. He, R. M. Gong, M. M. Zheng, and F. H. Huang, "Self-assembled phytosterol-fructose-chitosan nanoparticles as a carrier of anticancer drug," Journal of Nanoscience and Nanotechnology, vol. 13, no. 8, pp. 5935-5941, 2013.

[23] Y. Chen, J. Cao, H. Zhu et al., "Synthesis and evaluation of methionine and folate co-decorated chitosan self-assembly polymeric micelles as a potential hydrophobic drug-delivery system," Chinese Science Bulletin, vol. 58, no. 19, pp. 2379-2386, 2013.

[24] G. Unsoy, R. Khodadust, S. Yalcin, P. Mutlu, and U. Gunduz, "Synthesis of Doxorubicin loaded magnetic chitosan nanoparticles for $\mathrm{pH}$ responsive targeted drug delivery," European Journal of Pharmaceutical Sciences, vol. 62, pp. 243-250, 2014.

[25] K. I. David, L. R. Jaidev, S. Sethuraman, and U. M. Krishnan, "Dual drug loaded chitosan nanoparticles-sugar-coated arsenal against pancreatic cancer," Colloids and Surfaces B: Biointerfaces, vol. 135, pp. 689-698, 2015.

[26] K.-H. Park, D. Kang, and K. Na, "Physicochemical characterization and carcinoma cell interaction of self-organized nanogels prepared from polysaccharide/biotin conjugates for development of anticancer drug carrier," Journal of Microbiology and Biotechnology, vol. 16, no. 9, pp. 1369-1376, 2006.

[27] S. D. Weitman, A. G. Weinberg, L. R. Coney, V. R. Zurawski, D. S. Jennings, and B. A. Kamen, "Cellular localization of the folate receptor: potential role in drug toxicity and folate homeostasis," Cancer Research, vol. 52, no. 23, pp. 6708-6711, 1992.

[28] C. P. Leamon and J. A. Reddy, "Folate-targeted chemotherapy," Advanced Drug Delivery Reviews, vol. 56, no. 8, pp. 1127-1141, 2004. 
[29] H. Elnakat and M. Ratnam, "Distribution, functionality and gene regulation of folate receptor isoforms: implications in targeted therapy," Advanced Drug Delivery Reviews, vol. 56, no. 8, pp. 1067-1084, 2004.

[30] F. Alexis, E. Pridgen, L. K. Molnar, and O. C. Farokhzad, "Factors affecting the clearance and biodistribution of polymeric nanoparticles," Molecular Pharmaceutics, vol. 5, no. 4, pp. 505$515,2008$.

[31] S.-D. Li and L. Huang, "Pharmacokinetics and biodistribution of nanoparticles," Molecular Pharmaceutics, vol. 5, no. 4, pp. 496-504, 2008.

[32] H.-B. Tang, L. Li, H. Chen et al., "Stability and in vivo evaluation of pullulan acetate as a drug nanocarrier," Drug Delivery, vol. 17, no. 7, pp. 552-558, 2010.

[33] Y. Wang, H. Chen, Y. Liu et al., "PH-sensitive pullulan-based nanoparticle carrier of methotrexate and combretastatin A4 for the combination therapy against hepatocellular carcinoma," Biomaterials, vol. 34, no. 29, pp. 7181-7190, 2013.

[34] U. Kedar, P. Phutane, S. Shidhaye, and V. Kadam, "Advances in polymeric micelles for drug delivery and tumor targeting," Nanomedicine: Nanotechnology, Biology, and Medicine, vol. 6, no. 6 , pp. 714-729, 2010. 

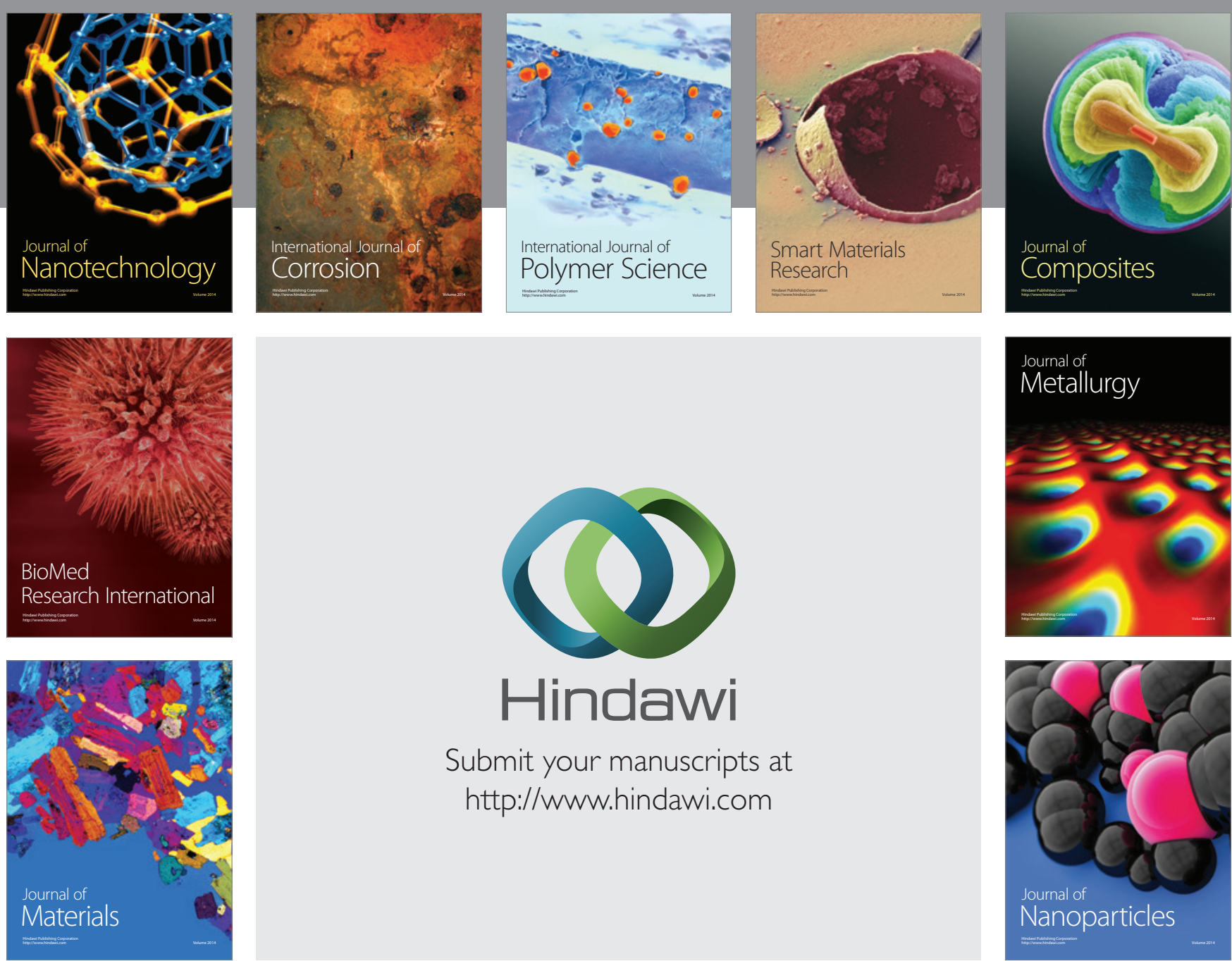

\section{Hindawi}

Submit your manuscripts at

http://www.hindawi.com

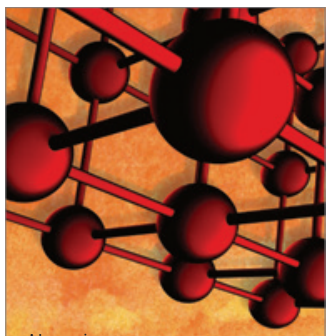

Materials Science and Engineering
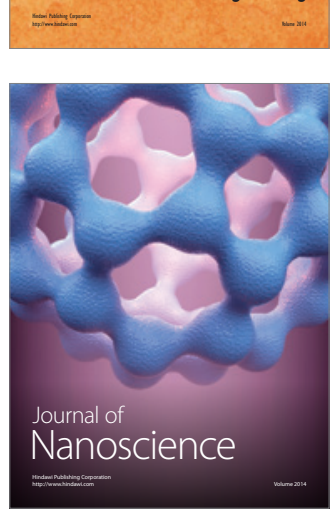
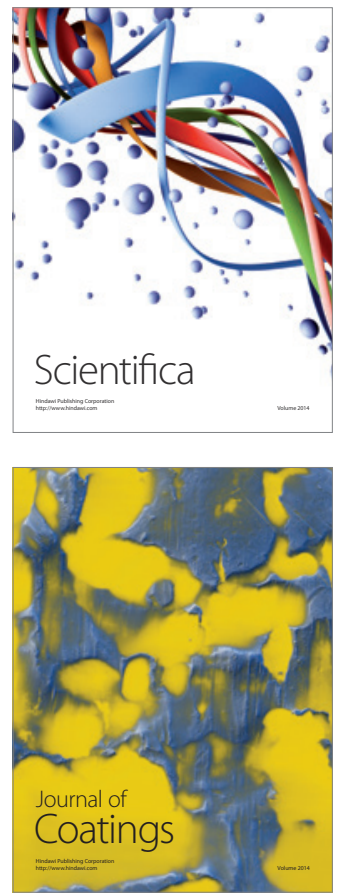
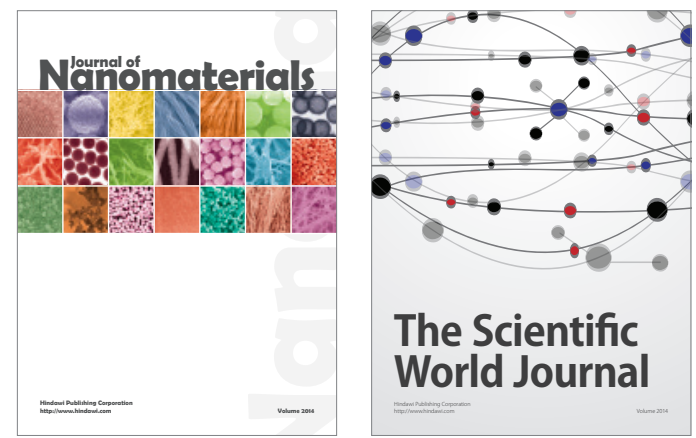

The Scientific World Journal
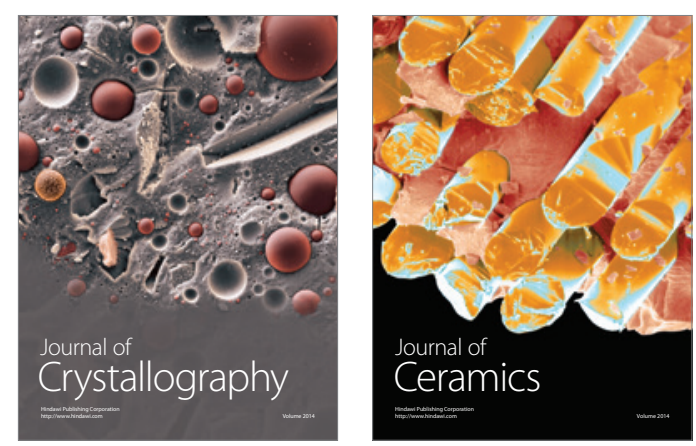
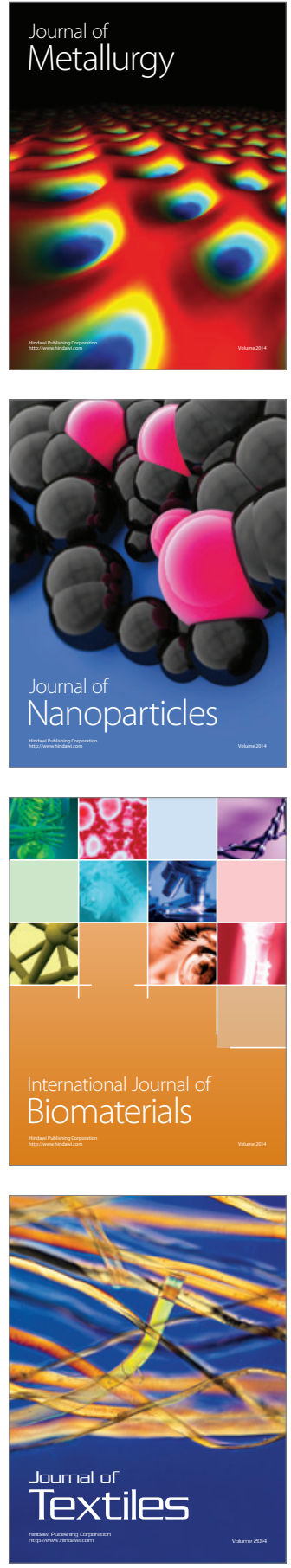